\title{
Types de formants dans la dérivation fonctionnelle polonaise et roumaine : bases théoriques
}

\author{
Types of formatives in Polish and Romanian functional \\ derivation: theoretical basis
}

\author{
Szymon Czarnecki \\ Uniwersytet im. Adama Mickiewicza w Poznaniu \\ szymon.czarnecki@amu.edu.pl
}

\begin{abstract}
The paper describes types of derivational formatives in Polish and Romanian functional, i.e. synchronic derivation, focusing on the proper derivation (i.e. derivation from single-word bases). The proper derivation is positioned as a type of word-formation and a branch of morphology. The author identifies the primary functions of derivational formatives: the semantic, pragmatic and syntactic function. The formatives are identified using a methodology developed in Polish word-formation theory, especially within nest derivatology. Applying a methodology developed for the description of the Polish word-formation system is aimed at distinguishing derivational formatives in Romanian. The author identifies and classifies the following types of derivational formatives in Romanian: affixes (among these prefixes, suffixes, postfixes and ambifixes), alternants (defined as members of alternation), negative formatives (in backformation), paradigmatic formatives (defined as changes in inflexional paradigm), zero formative and accentual formatives. The author also suggests including the types of word-formation stems in the description of the formal structure of derivatives.
\end{abstract}

Keywords: word-formation, derivation, morphology, formative, derivational stem, derivatology, Romanian

\section{INTRODUCTION}

L'objectif de cet article est de distinguer les types de formants dérivationnels théoriquement possibles ainsi que les types de structures formelles des mots dérivés dans la dérivation fonctionnelle de la langue roumaine. Cet objectif sera atteint grâce 
à l'examen de l'ensemble de types de formants décrits par la linguistique polonaise, suivi de la vérification de leur occurrence potentielle en roumain, ainsi qu'à l'aide des solutions méthodologiques appartenant à l'auteur même ${ }^{1}$. Nous voyons la justification de cette procédure dans le fait que l'approche que nous utilisons fait partie d'un appareil conceptuel plus large, employé dans les recherches sur la dérivation roumaine et emprunté à la linguistique polonaise. Quant aux résultats de cet article, nous voyons également d'autres possibilités de leurs applications ultérieures dans des études contrastives. Un objectif supplémentaire, mais non pas moins important, est de contribuer à l'internationalisation de la discipline ${ }^{2}$, alors que le choix de la méthode, appliquée antérieurement à l'analyse de la langue polonaise, dans le but de décrire une langue non-slave est traité aussi comme un essai de falsification.

\section{Méthodologie et appareil notionnel}

La méthodologie choisie de nous a été développée dans le cadre de la dérivatologie polonaise, en particulier dans celui de la méthode «nidologique » (Skarżyński, 1999 et 2003). Aussi bien les définitions du mot motivé et du mot motivant que la méthode même de déterminer la direction de la dérivation sont empruntées respectivement à Grzegorczykowa et Puzynina (1998, pp. 361-364) et à Kaproń-Charzyńska (2005, pp. 55-57) - à cette exception près que, pareillement à Vogelgesang et Jadacka, nous renonçons à la notion de multimotivation (Vogelgesang, 2001, pp. 26-28; Jadacka, 2003 p. 37). La définition du formant dérivationnel comme exposant formel de la dérivation, constituant la différence entre le dérivé et la base dérivationnelle, est empruntée, à son tour, à Grzegorczykowa et Puzynina (1998, p. 363) - il faut préciser quand même que la différence en question ne se manifeste pas dans seules les propriétés formelles, mais aussi dans les propriétés morphosyntaxiques. Dans le cadre des recherches visant à distinguer avant tout les formants simples, nous renonçons à la notion de formant complexe pour la remplacer par la notion de cooccurrence des formants ${ }^{3}$.

Nous proposons de nommer le type de formation des mots que nous avons choisi pour cette étude - dérivation fonctionnelle (ou sémantico-formelle). Le premier terme a été trouvé dans l'ouvrage de Nagórko (2010, p. 183), et le second, synonyme, - dans celui de Grzegorczykowa et Puzynina (1998, p. 361). Dans son cadre, nous distinguons la dérivation propre, c'est-à-dire celle qui se réfère aux

\footnotetext{
${ }^{1}$ Il s'agit avant tout de l'inclusion des types de thèmes dérivationnels dans la description de la structure formelle des dérivés.

${ }^{2}$ La nécessité d'une internationalisation des recherches sur la formation des mots est soulignée par Müller, Ohnheiser, Olsen et Rainer (2015, p. VIII).

${ }^{3}$ Cela implique également l'utilisation de la notion de co-formant.
} 
dérivés simples (non-composés) (Grzegorczykowa, Puzynina, 1998, p. 36). Nous pensons que l'emploi d'une telle terminologie est nécessaire, car la dérivation sémantico-formelle (fonctionnelle), qui est un sous-domaine de la morphologie, n'est pas un seul type possible de formation synchronique des mots.

\section{Autres principes}

Nous sommes persuadés que, pour les besoins d'une description correcte et effective de la dérivation, il est nécessaire de faire une distinction nette des notions suivantes : 1) les processus dérivationnels et leurs résultats (formants) : si la suffixation est un processus dérivationnel et que son résultat est un suffixe, le terme « alternance » se rapporte pareillement à un processus tandis que le résultat de celui-ci est un alternant ; 2) les exposants de la dérivation (formants), eux-mêmes, et leurs fonctions, c'est-à-dire des tâches que les formants accomplissent.

\section{Trois fonctions des formants primaires}

Nous trouvons très utile la distinction de trois fonctions primaires des formants : la première consistant en changements syntaxiques, la deuxième consistant en changements sémantiques, et la troisième - en changements pragmatiques du dérivé par rapport à sa base. Cette classification s'appuie sur les affirmations de J. Kuryłowicz (1936 ; cité par Grzegorczykowa, Puzynina, 1998, p. 375). Cependant, nous décomposons la «fonction sémantique » qu'il a discernée, en excluant de celle-ci la fonction pragmatique comme distincte, car dans le cas de certains dérivés (par exemple les hypocoristiques ou les péjoratifs), il est difficile de parler d'un changement sémantique des dérivés par rapport à la base. Le référent y reste le même, seule l'attitude du locuteur face à la réalité change, et, par conséquent, il s'agit là du domaine de la pragmatique, et non pas de la sémantique.

Les trois fonctions primaires permettent de distinguer les formants primaires des ceux qui sont secondaires, c'est-à-dire de tels formants qui remplissent une quatrième fonction qui est de valeur secondaire, elle aussi. Celle-ci consiste en l'adaptation de la base dérivationnelle à l'adhésion des formants primaires qui sont, eux, les exposants proprement dits des différences syntaxiques, sémantiques et pragmatiques.

Cela permet aussi de distinguer les alternants conditionnés phonologiquement (en tant que formants secondaires) des alternants qui sont des exposants de différences de sens (en tant que formants primaires) et, par conséquent, de distinguer la dérivation sémantico-formelle et d'autres types de formations des mots qui ne rendent compte d'aucune des trois fonctions primaires ou de changements morphosyntaxiques possibles. Il convient de noter ici que les formants primaires peuvent être polyfonctionnels. 
4. La dérivation sémantico-formelle en tant que type de formation des mots sensu lato ${ }^{4}$

Nous pensons qu'il est nécessaire de mieux définir les limites de la dérivation sémantico-formelle en tant que sous-domaine de la morphologie ${ }^{5}$ aussi bien que la place qu'elle occupe par rapport aux autres types de formation des mots, tout en indiquant les phénomènes qui entrent dans son champ d'application et ceux qui n'y entrent pas.

$\mathrm{Au}$ moins une partie des processus qui font l'objet d'autres types de formation des mots sont également de nature synchronique ou du moins contemporaine, ce qui permet aux sujets parlants d'en prendre conscience. Cela se réfère aux processus de : [1] la pseudo-dérivation qui est l'adaptation des emprunts, p. ex. par la suffixation des mots étrangers, comme dans la formation roum. șerui $i_{\mathrm{VB}}$ 'partager sur un réseau social', de l'angl. to share $\mathrm{VB}_{\mathrm{VB}}$ 'idem' (cf. Viaro, Bizzocchi, 2016); [2] la dérivation « condensationnelle » (cf. Kreja, 1996, cité par Vater, 2015, p. 99), y compris l'acronymie, la troncation et le télescopage (formation des mots-valises) ; [3] la dérivation sémantique, c'est-à-dire la dérivation synchronique de nouvelles significations de mots déjà existants sans recours aux exposants formels, à moins qu'il n'y ait pas de changements dans les fonctions syntaxiques du mot, comme par exemple dans la paire roum. minister $r_{\mathrm{SN}}$ 'ministère (institution)' $\rightarrow$ minister $_{\mathrm{SN}}$ 'ministère (bâtiment)', ou angl. crane $_{\mathrm{S}}$ 'grue (oiseau)' $\rightarrow$ crane $_{\mathrm{S}}$ 'grue (de chantier)', où la métonymie entre en jeu (cf. Apresjan, 2000); [4] la dérivation phraséologique, c'est-à-dire la formation d'unités lexicales comprenant plusieurs mots pour désigner un seul référent).

Le statut d'objet de la dérivation sémantico-formelle ne peut pas être attribué non plus à la création extra-grammaticale et arbitraire de nouveaux mots (comme dans l'exemple angl. quark $k_{\mathrm{S}}$ 'quark'), c'est-à-dire à la nomination primaire (cf. Lachur, 2004, p. 161), même si de tels mots ont été créés plus ou moins sur la base de mots déjà existants, comme dans le cas des exemples : angl. radar ${ }_{\mathrm{S}}$ 'radar' ou pol. polon $_{\mathrm{SM}}$ 'polonium (un élément chimique nommé en l'honneur du pays d'origine de Marie Curie)'. Cette prémisse ne nie pas l'existence de dérivés associatifs (onomasiologiques), à condition que les relations formelles soient toutefois maintenues et que la périphrase dérivationnelle puisse être formulée à l'aide des caractéristiques non définitoires du référent : roum. zori $i_{\mathrm{SM} \text {.PL-TANT }}$ 'aube' $\rightarrow$ zorea $_{\mathrm{SF} . \mathrm{SG}}$ 'ipomée, espèce de plantes herbacées dont les fleurs s'ouvrent le matin', pol. lipa $a_{\mathrm{SF}}$ 'tilleul' $\rightarrow$ lipiec $_{\mathrm{SM}}$ 'juillet; le mois où les tilleuls fleurissent'.

\footnotetext{
${ }^{4}$ C'est-à-dire la formation de nouveaux lexèmes en général (Vater, 2015, p. 84).

${ }^{5}$ L'adjectif « formel » renvoie ici aux moyens morphologiques dont rend compte ce type de formation des mots.
} 
En raison de la similitude des processus (utilisation fréquente des mêmes formants, maintien des rapports formels et sémantiques de la base dérivationnelle avec le mot dérivé), nous considérons comme appartenant au domaine de la dérivation sémantico-formelle également les sous-domaines suivants : [1] la composition formation des mots composés, c'est-à-dire des lexèmes motivés et fondés par plusieurs bases dérivationnelles, p. ex. : roum. polono-român $n_{\mathrm{ADJ}}$ 'polonais-roumain', et [2] l'univerbisation - formation des mots nouveaux qui sont sémantiquement motivés par plusieurs mots sources à la fois, mais qui sont fondés par un seul d'entre eux, que ce soit par suffixation, comme dans pol. stare $_{\mathrm{ADJ}}$ miasto $_{\mathrm{SN}}$ 'cité (vieille ville)' $\rightarrow$ starówk $a_{\mathrm{SF}}$ 'idem', ou par ellipse et substantivation, comme dans pol. $r z u t_{\mathrm{SM}}$ karny $_{\mathrm{ADJ}}$ 'tir de pénalité' $\rightarrow$ karny $_{\mathrm{SM}}$ 'idem', cf. roum. bara $a_{\mathrm{SF}}$ transversală $\check{\mathrm{ADJ}}_{\mathrm{DJ}}$ 'barre transversale' $\rightarrow$ transversala $\breve{S}_{\mathrm{SF}}$ 'idem'. Nous considérons ces derniers comme des dérivés simples en raison de l'impossibilité de les distinguer nettement des dérivés sémantiquement irréguliers (dans lesquels le sens de la base et le sens du formant n'englobe pas tout le sens lexical du dérivé, cf. pol. sernik $k_{\mathrm{SM}}$ 'gâteau au fromage' $\leftarrow s e r_{\mathrm{SM}}$ 'fromage', cf. Grzegorczykowa, Puzynia, 1998, p. 372). Prise en compte l'économie de l'exposition, nous nous concentrerons sur la dérivation propre, en ne faisant appel à la composition qu'occasionnellement.

\section{Types de formants vs types de thèmes dérivationnels}

La méthode choisie de nous prévoit que la structure formelle du dérivé comporte deux éléments : l'un d'eux est le thème de la base dérivationnelle, l'autre est le formant (ou les formants). Il faut souligner à ce propos qu'une seule et simple identification des formants qui participent à la dérivation est insuffisante pour une description correcte et complète des structures formelles que réalisent les dérivés.

Comme l'a démontré I. Coteanu (2007, pp. 14-15), on trouve en roumain des mots dérivés des formes flexionnelles du mot motivant autres que le nominatif singulier sans article, cf. :

$\operatorname{colț(}(\varnothing)_{\mathrm{SN} . \mathrm{SG}}\left(\right.$ colţuri $\left._{\mathrm{PL}}\right)$ 'angle' $\rightarrow$ colţur-os $(\varnothing)_{\mathrm{ADJ}}$ 'anguleux'

$m o f t(\varnothing)_{\mathrm{SN} . S \mathrm{G}}\left(\right.$ moftur $\left._{\mathrm{PL}}\right)$ 'caprice' $\rightarrow$ moftur-os $(\varnothing)_{\mathrm{ADJ}}$ 'capricieux'

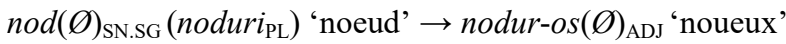

On peut en déduire que le suffixe $-o s(\varnothing)$ est attaché à la forme plurielle du substantif colț(uri), ce qui est accompagné de la chute de l'élément final (-i). Pour une description correcte de la structure formelle du dérivé, il est nécessaire d'y inclure non seulement les types de formants, mais aussi les types de thèmes dérivationnels ; dans le dernier cas analysé, il s'agit d'un thème secondaire (avec la désinence du pluriel). Dans le cas contraire, si nous nous limitons à seule l'analyse $\mathrm{du}$ formant, nous obtenons un faux résultat : colțos. Un tel adjectif existe aussi, mais 
il a un sens différent et provient d'un lexème différent. De plus, le dérivé maintient le sens du pluriel propre à la forme flexionnelle dont il est issu, et sa paraphrase roumaine 'care are colțuri' en témoigne (Coteanu, 2007, p. 14). Nous considérons que ce fait - le maintien des valeurs catégorielles d'une forme flexionnelle de base dans la sémantique du dérivé - constitue l'argument décisif pour l'incorporation des types de thèmes, sur les mêmes principes que les types de formants, dans la description des structures formelles des mots dérivés.

Le nombre plus grand de dérivés fondés sur des formes flexionnelles autres que la forme de base (l'infinitif, p. ex.), héritant le sens catégoriel de ces dernières, se retrouve dans la classe des verbes roumains, cf. :

$c a ̆ d e a_{\mathrm{VB.INF}}\left(c a ̆ z a ̂ n d_{\mathrm{PTCP} . \mathrm{ACT}}\right)$ 'tomber' $\rightarrow$ căz-ător $(\varnothing)_{\mathrm{ADJ}}$ 'tombant'

sparge $_{\mathrm{VB.INF}}\left(\right.$ spărgând $\left.{ }_{\mathrm{PTCP.ACT}}\right)$ 'cambrioler' $\rightarrow$ spărg-ător $(\varnothing)_{\mathrm{SM}}$ 'cambrioleur'

frânge $_{\mathrm{VB} . \mathrm{INF}}\left(\right.$ frânt $\left._{\mathrm{PTCP.PASS}}\right)$ 'casser' $\rightarrow$ frânt-ur $(\breve{a})_{\mathrm{SF}}$ 'casse (débris)' (Coteanu, 2007, p. 15)

Les exemples ci-dessus démontrent que les dérivés proviennent respectivement du thème du participe actif $c \breve{a z}$ - et du thème du participe passif frânt ${ }^{6}$. Les processus dérivationnels dans les deux paires, ainsi que dans la formation de colțuros $_{\mathrm{ADJ}}$, sont la suffixation et le changement du paradigme flexionnel. Il faut donc que la structure formelle de ces dérivés soit décrite comme suit :

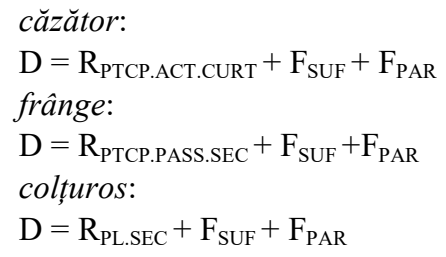

\section{TYPES DE FORMANTS}

\section{Formants affixaux}

L'un des types de formants les plus fréquents dans la dérivation des deux langues sont les affixes dérivationnels. Les affixes et leurs sous-types peuvent être déterminés en tenant compte des critères suivants :

1) leur position par rapport au thème de la base dérivationnelle; les préfixes sont les affixes qui précèdent le thème de la base dérivationnelle, ceux qui le suivent sont, à leur tour, les suffixes ; ensemble, ces deux sous-types constituent le groupe des adfixes; les infixes sont les affixes qui se trouvent à l'intérieur du thème de la base dérivationnelle ;

\footnotetext{
${ }^{6}$ Les notions de « catégories flexionnelles » et de «types de thèmes » du verbe roumain proviennent d'une description exhaustive de la conjugaison roumaine (Cychnerski, 1999).
} 
2) la position par rapport aux désinences du dérivé ou à son thème secondaire ; les extrafixes se situent « en dehors » de la forme du mot: [a] les postfixes se placent après les désinences, [b] les antéfixes apparaissent avant le thème de la base dérivationnelle, en conservant un certain degré d'autonomie ;

3) la continuité ou la discontinuité formelle ; les circonfixes sont des affixes discontinus qui se placent généralement des deux côtés du thème de la base dérivationnelle, constituant ainsi une sorte d'adffixes ;

4) la position fixe ou la mobilité ; les ambifixes, ou les affixes mobiles, sont ceux dont la position varie selon les différentes formes flexionnelles du même lexème (Mugdan, 2015).

En polonais ainsi qu'en roumain, les affixes les plus nombreux sont les suffixes. Nous citons ci-dessous les exemples où le suffixe est un formant dérivationnel :

roum. aduna $a_{\mathrm{VB}}$ 'assembler' $\rightarrow$ aduna-r $(e)_{\mathrm{SF}}$ 'assemblement'

roum. $\operatorname{bot}(\varnothing)_{\mathrm{SN}}$ 'museau' $\rightarrow$ bot-niț(ă $)_{\mathrm{SF}}$ 'muselière'

roum. istori $(e)_{\mathrm{SF}}$ 'historie' $\rightarrow$ istor-ic $(\varnothing)_{\mathrm{ADJ}}$ 'historique'

pol. płaszcz $(\varnothing)_{\mathrm{SM}}$ 'manteau' $\rightarrow$ ptaszcz-yk(Ø) $)_{\mathrm{SM}}$ 'idem (diminutif)'

Les deux langues ont également un certain nombre de préfixes, cf. :

roum. aşteptat $(\varnothing)_{\mathrm{ADJ}}$ 'attendu' $\rightarrow$ ne-aştepat ${ }_{\mathrm{ADJ}}$ 'inattendu'

roum. incepe $e_{\mathrm{VB}}$ 'commencer' $\rightarrow$ re-începe $e_{\mathrm{VB}}$ 'recommencer'

roum. arma $_{\mathrm{VB}}$ 'armer' $\rightarrow$ dez-arma $a_{\mathrm{VB}}$ 'désarmer'

pol. gorliw $(y)_{\mathrm{ADJ}}$ 'zélé' $\rightarrow$ nad-gorliwy ${ }_{\mathrm{ADJ}}$ 'trop zélé'

Les postfixes sont très rares dans les deux langues. En voici quelques exemples :

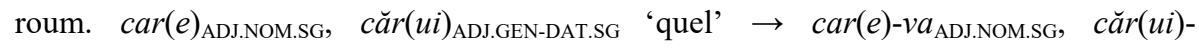



roum. $c \hat{t} t(i)_{\text {ADJ.M.NOM.PL }}$ cât(or $)_{\text {ADJ.GEN-DAT.PL }}$ 'combien $(\mathrm{de})$ ' $\rightarrow$ câțt(i)-va $a_{\text {ADJ.M.NOM.PL }}$,



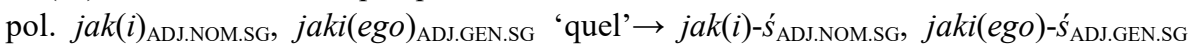
'quelconque'

Les circonfixes et les infixes en polonais ont été identifiés par Nagórko (2010, pp. 91-92), cf. :

$\operatorname{wod}(a)_{\mathrm{SF}}$ 'eau' $\rightarrow$ na-wod-n(ić) $)_{\mathrm{VB} . P F V}$ 'irriguer' polsk $(i)_{\mathrm{ADJ}}$ 'polonais' $\rightarrow$ po polsk- $u_{\mathrm{ADV}}$ 'en polonais, à la polonaise'

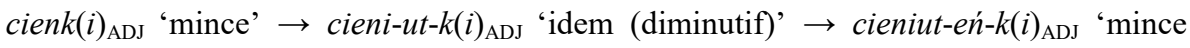
(diminutif du second degré)'

Les circonfixes n'existent probablement pas en roumain. La parasynthèse, c'est-à-dire la formation (p. ex.) des verbes au moyen de la préfixation et de la suffixation en même temps, ne peut pas être considérée comme circonfixation parce 
que la condition nécessaire que doit remplir un circonfixe est son intégrité sémantique et fonctionnelle. Dans les formations du type $f i(u)_{\mathrm{SM}}$ 'fils' $\rightarrow \hat{\imath} n-f i-a_{\mathrm{VB}}$ 'adopter' le segment - $a$ est, du point de vue dérivationnel, un suffixe thématique du verbe et n'apparait pas dans toutes les formes flexionnelles de ce lexème, cf. înfiez 'j'adopte'.

La présence des infixes en roumain est discutable. Comme une certaine prémisse pour leur identification peut être considérée l'analyse de I. Coteanu (2007, p. 29) qui dérive la formation poduleț $t_{\mathrm{SN}}$ de poduț $_{\mathrm{SN}}$; les deux formes sont des diminutifs du mot $\operatorname{pod}_{\mathrm{SN}}$ 'pont'. Cependant, le segment -ul- ne peut être interprété comme infixe que dans le cas où il existe un rapport de gradation entre les deux diminutifs, comme

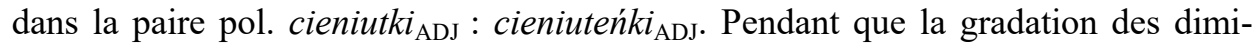
nutifs, elle-même, est possible en roumain (cf. casă $\breve{S F}_{\mathrm{SF}}$ 'maison' $\rightarrow$ căs-uț( $(\breve{a})_{\mathrm{SF}}$ 'maisonnette' $\rightarrow$ căs-cioar $(\breve{a})_{\mathrm{SF}}$ 'maisonnette (diminutif du second degré)'), les dictionnaires roumains définissent poduleț $t_{\mathrm{SN}}$ comme synonyme de poduț $_{\mathrm{SN}}$. Il est évident donc que des recherches supplémentaires sont nécessaires dans ce domaine.

En ce qui concerne les autres types de formants, l'affixe się est considéré le plus souvent comme un postfixe dans la littérature polonaise. Cependant, comme le soulignent Grzegorczykowa et Puzynina (1998, p. 367), il a un caractère mobile : il peut apparaître aussi bien après les désinences (c'est-à-dire dans les positions postfixales) qu'avant le thème du mot, tout en conservant alors une certaine autonomie (donc dans les positions antéfixales). Nous proposons alors de le traiter comme un ambifixe. Puisque les positions qu'il occupe peuvent être considérées, en généralisant les faits, comme extrafixales, il appartient aussi à la classe des extrafixes.

L'affixe roumain se démontre les mêmes propriétés dans la structure des verbes pronominaux. Dans certaines formes flexionnelles, il apparaît avant le thème du verbe en conservant son autonomie, cf. se mai îndoiește '(il) doute encore' ; dans $\mathrm{d}$ 'autres, il apparaît après le thème : îndoindu-Se $e_{\mathrm{PTCP.ACT}}$ 'doutant'.

\section{Les alternants conditionnés phonologiquement et le problème des intermorphes et des affixes composés}

Dans la littérature polonaise, nous découvrons la notion de segments dépourvus de sens. Ceux-ci peuvent apparaître entre deux morphèmes et n'appartenir à aucun d'entre eux (certains auteurs les nomment " connectifs » ou " intermorphes »). Ils peuvent aussi constituer de segments dépendants des affixes élargis (étant appelés alors « submorphes »; cf. Nagórko, 2010, p. 87 ; Grzegorczykowa i Puzynina, 1998, p. 367). Cependant, l'incorporation des segments privés de signification dans la classe des morphèmes entraîne des problèmes de délimitation de ces derniers (Laskowski, 1998, pp. 73-74). Nagórko analyse pol. dar ${ }_{\mathrm{SM}}$ 'cadeau' comme un dérivé de 
$d a c_{\mathrm{VB}}$ 'donner' avec le suffixe -ar (comme dans pol. such $(y)_{\mathrm{ADJ}}$ 'sec' $\rightarrow$ such$\operatorname{ar}(\varnothing)_{\mathrm{SM}}$ 'biscotte') et la superposition de morphèmes : $d a$ - + -ar (Nagórko, 2010, p. 87). Pourquoi alors, en supposant l'existence d'intermorphes, ne devrait-on pas considérer que c'est seulement $-r$ qui est le suffixe dans les deux formations, et le segment - $a$ - est un intermorphe dans le mot such-a-r? L'admission de l'existence de segments intermorphémiques privés de signification à l'intérieur d'une morphologie basée sur la notion du morphème défini comme la plus petite unité de sens pose plus de problèmes qu'elle n'en résout.

Les affixes élargis - enrichis d'un submorphe doivent provenir du processus d'absorption morphologique, c'est-à-dire de l'absorption d'un élément de la base dérivationnelle par l'affixe, à la suite d'une double division du dérivé, comme dans les exemples polonais ci-dessous (Grzegorczykowa, Puzynina, 1998, p. 366) :

$\operatorname{druk}(\varnothing)_{\mathrm{SM}}$ 'impression' $\rightarrow \operatorname{druk-arz}(\varnothing)_{\mathrm{SM}}$ 'imprimeur' $\rightarrow \operatorname{drukar-stw}(o)_{\mathrm{SN}}$ 'imprimerie'



Il est à remarquer que l'absorption morphologique consiste d'habitude en l'absorption d'un affixe par un autre affixe. En polonais, l'absorption au-delà des limites d'un seul nid dérivationnel est également possible, ainsi que l'absorption du segment final d'un autre morphème (le thème de la base dérivationnelle, le plus souvent) :

kierow $(a c)_{\mathrm{VB}}$ 'conduire' $\rightarrow$ kierow-c $(a)_{\mathrm{SM}}$ 'chauffeur, conducteur'

$\operatorname{zwiad}(\varnothing)_{\mathrm{SM}}$ 'éclairage (militaire)' $\rightarrow$ zwiad-owc $(a)_{\mathrm{SM}}$ 'éclaireur' ${ }^{7}$

$\operatorname{Indi}(e)_{\mathrm{NP.SN}-\mathrm{M} . \mathrm{PL}-\mathrm{TANT}} / \mathrm{indj} \varepsilon /$ 'Inde' $\rightarrow$ ind $[y] j-s k(i)_{\mathrm{ADJ}}$ 'indien'

$\operatorname{Kong}(o)_{\mathrm{NP} . \mathrm{SN} .}$ 'Congo' $\rightarrow$ kongijsk $(i)_{\mathrm{ADJ}}$ 'congolais'

Chile $_{\mathrm{NP} . \mathrm{SN} . \mathrm{INV}} \rightarrow{\text { chilijsk }(i)_{\mathrm{ADJ}} \text { 'chilien, }}^{, 8}$

Les segments décrits ci-dessus comme des « affixes élargis » peuvent être considérés comme affixes, à condition qu'il soit possible de démontrer les différences de signification ou de capacités d'adhésion entre eux et leurs équivalents « simples » (ce qui dépend du sens ou de l'appartenance catégorielle de la base dérivationnelle). Le suffixe -owc(a), par exemple, contribue à la formation des substantifs dénominaux qui expriment l'agent, surtout quand le verbe correspondant n'existe pas. Une différence pareille de signification ou de capacités d'adhésion ne peut être toutefois démontrée dans le cas d'un prétendu affixe -ijsk(i) en comparaison avec sa forme «simple »-sk(i), il n'y a donc aucune raison de le considérer comme la réalisation d'un morphème distinct. Cependant, il est possible d'interpréter le segment - $i j$ - comme un type de formant différent de celui de l'affixe, ce qui résulte d'autres façons de diviser le mot en morphes.

\footnotetext{
${ }^{7}$ Zołotowa (1962, pp. 319-320).

${ }^{8}$ Intentionnellement, nous ne divisons pas les morphèmes des deux derniers dérivés.
} 
Si nous constatons que l'origine de ce phénomène réside dans le thème (son incapacité d'adhésion de l'affixe), et à la suite de cette prémisse on obtient per analogiam un effet sous la forme d'un segment supplémentaire aussi à partir du thème appartenant à la (une autre) base dérivationnelle (qui réalise le même type dérivationnel, $\mathrm{cf}$. Indi(e) $\rightarrow$ indyj-sk(i)), la solution alternative s'impose d'elle-même. Rien n'empêche d'admettre qu'aussi ce moyen-ci de résoudre le problème d'incapacité d'adhésion fasse, en tant que tel, partie du thème, en constituant donc un exemple du type de formant interne - par analogie avec la flexion interne (et par opposition aux formants externes, c'est-à-dire les affixes). Une telle approche laisse traiter ce processus dérivationnel tout simplement comme une alternance du thème (alternance quantitative positive), ce qui ne prive pas l'alternant de ses propriétés d'adhésion, mais enlève pourtant au segment analysé le statut d'unité morphologique indépendante.

Le segment -ij- est donc un élargissement du thème, et par conséquent, le membre alternant conditionné phonologiquement (l'autre membre de l'alternance étant le zéro). Les morphes Kong- et kongij- sont des allomorphes du morphème KONG-. L'ultime segment, considéré comme un thème dérivationnel, est le type particulier de celui-ci, à savoir un thème élargi ou combinant (appelé aussi composant, car le phénomène identique se produit également dans les mots composés, cf. româno-france $z_{\mathrm{ADJ}}$ 'roumain-français'" (cf. Mugdan, 2015, pp. 268-270). L'inclusion du segment -ij- dans le thème kongij- de la base dérivationnelle est de même motivée par le fait que la même alternance se produit dans d'autres dérivés de la même base, avant d'autres suffixes, cf. Kongijczy $k_{\mathrm{SM}}$ 'Congolais (personne)'. C'est un type spécifique d'allomorphisme, réalisé non pas dans le paradigme flexionnel du lexème, mais dans son paradigme dérivationnel. Cette interprétation simplifie la description de la structure de nombreux dérivés, par exemple dans le cas de Chile $e_{\mathrm{SN}}$ $\rightarrow$ chilijski $_{\mathrm{ADJ}}$, on peut parler d'une alternance mixte quantitative et qualitative $e: i j$ au lieu d'y voir la chute de -e et l'adhésion de -ij-.

Nous pouvons décrire le processus ainsi défini au moyen de la formule suivante (pour rendre la formule plus claire, les mots membres de chaque paire sont représentés phonologiquement) :



La structure même du dérivé kongijski composée de deux parties (type de thème + type(s) de formant) peut être décrite à l'aide de la formule suivante :

$$
\mathrm{D}=\mathrm{R}_{\mathrm{EXT}}+\mathrm{F}_{\mathrm{ALT}}, \mathrm{F}_{\mathrm{ALT}}, \mathrm{F}_{\mathrm{SUF}}, \mathrm{F}_{\mathrm{PAR}}
$$

\footnotetext{
${ }^{9}$ Une telle interprétation résout également le problème de l'interfixe en tant qu'élément asémantique de mots composés. En plus, elle s'appuie aussi sur le fait que les interfixes ou les intermorphes semblent d'habitude être phonologiquement plus liés au premier segment du dérivé, cf. /kənjijțīik/ (cf. Mugdan, 2015, pp. 268-270). En outre, c'est l'orthographe qui mène aux pareilles conclusions - puisqu'on écrit româno-francez, et non pas *român-ofrancez.
} 
Un pareil élargissement du thème de la base dérivationnelle avant le suffixe, interprété par analogie avec d'autres paires dérivationnelles, se retrouve aussi en roumain, cf. :

fovism $(\varnothing)_{\mathrm{SN}}$ 'fauvisme' $\rightarrow$ fov- $\varnothing$-ist $(\varnothing)_{\mathrm{ADJ}}$ 'fauviste'

Dinamo $_{\mathrm{NP} . \mathrm{SN}}($ București $) \rightarrow$ dinamov-ist $(\varnothing)_{\mathrm{SM}}$ 'joueur ou supporter de « Dinamo

București »'

Congo $_{\mathrm{NP} . \mathrm{SN}} \rightarrow$ congol-ez $(\varnothing)_{\mathrm{SM}}{ }^{\text {'Congolais' }}$

La description des formations de type dinamovist $_{\mathrm{SM}}$ se fait de la même façon que celle des formations polonaises de type kongijski $i_{\mathrm{ADJ}}$ :

$$
\begin{aligned}
& \mid \text { dinamo : dinamov } \mid(\varnothing) \rightarrow \operatorname{dinamo}[\mathrm{v}]-\operatorname{ist}(\varnothing) \\
& \mathrm{D}=\mathrm{R}_{\mathrm{EXT}}+\mathrm{F}_{\mathrm{ALT}}, \mathrm{F}_{\mathrm{SUF}}, \mathrm{F}_{\mathrm{PAR}}, \mathrm{F}_{\mathrm{ACC}}
\end{aligned}
$$

Les alternances du thème avant le suffixe peuvent également avoir un caractère contraire en polonais : la réduction du thème au lieu de son élargissement, par exemple dans la paire : Egipt $(\varnothing)_{\mathrm{NP} . \mathrm{SM}}$ 'Égypte' $\rightarrow$ egip-sk $(i)_{\mathrm{ADJ}}$ 'égyptien'. La formation egipski $i_{\mathrm{ADJ}}$ va avoir la description suivante :

$$
\begin{aligned}
& |\{\varepsilon j i p t: \varepsilon j i p \widehat{t 6}\}:\{\varepsilon j i p\}|(\varnothing) \rightarrow \text { ejip[Ø]-sc(i) } \\
& \mathrm{D}=\mathrm{R}_{\mathrm{RED}}+\mathrm{F}_{\mathrm{ALT}}, \mathrm{F}_{\mathrm{SUF}}, \mathrm{F}_{\mathrm{PAR}}
\end{aligned}
$$

Des formations similaires existent aussi en roumain :

cronologi $(e)_{\mathrm{SF}}$ 'chronologie' $\rightarrow$ cronolog $[\varnothing]-i s t(\varnothing)_{\mathrm{SM}}$ 'chronologiste'

ecologi $(e)_{\mathrm{SF}}$ 'écologie' $\rightarrow \operatorname{ecolog}[\varnothing]-i s t(\varnothing)_{\mathrm{SM}}$ 'écologue'

Chile $_{\mathrm{NP} . \mathrm{SN} . \mathrm{INV}}$ 'Chili' $\rightarrow \operatorname{chil}[\varnothing]-\operatorname{ian}(\varnothing)_{\mathrm{ADJ}}$ 'Chilien'

La notion d'alternances quantitatives négatives conditionnées phonologiquement peut également être étendue aux cas où les alternances se produisent avant un autre type d'affixe(s), à savoir avant l'ensemble tout entier de désinences qui est soumis à des changements propres au formant paradigmatique, cf. :

roum. ecologi $(e)_{\mathrm{SF}}$ 'écologie' $\rightarrow$ ecolog $[\varnothing](\varnothing)_{\mathrm{SM}}$ 'écologue'

roum. biologi $(e)_{\mathrm{SF}}$ 'biologie' $\rightarrow$ biolog $[\varnothing](\varnothing)_{\mathrm{SM}}$ 'biologiste'

pol. ekologi $(a)_{\mathrm{SF}} / \varepsilon k$ logja/ 'écologie' $\rightarrow e k o l o g[\varnothing](\varnothing)_{\mathrm{SM}}$ 'écologue'

Il est également possible de trouver les suffixes dits élargis dans la langue roumaine : ils sont créés par l'absorption d'autres suffixes ou d'une partie finale appartenant au thème d'autres bases dérivationnelles.

apuca $_{\mathrm{VB}}\left(\right.$ apucat $\left._{\mathrm{PTCP.PASS}}\right)$ 'prendre' $\rightarrow$ apucăt-ur $(\breve{a})_{\mathrm{SF}}$ 'prise'

$c a ̆ d e a_{\mathrm{VB}}\left(c a ̆ z u t_{\mathrm{PTCP} . \mathrm{PASS}}\right)$ 'tomber' $\rightarrow$ căz-ătur $(\breve{a})_{\mathrm{SF}}$ 'chute'

șterge $_{\mathrm{VB}}\left(\right.$ șters $\left._{\mathrm{PTCP} . \mathrm{PASS}}\right)$ 'effacer' $\rightarrow$ șters-ătur $(\breve{a})_{\mathrm{SF}}$ 'effacement' 
Comme l'observe I. Coteanu (2007, p. 38), les dérivés qui se terminent par -tură proviennent le plus souvent du thème secondaire participial du verbe, en se construisant à l'aide du suffixe -ur( $\breve{a})$. Toutefois, dans les cas où le participe se termine par $-s$ ou $-u t$, il se produit une suffixation à l'aide de -ătur $(\breve{a})$; cette limitation distributionnelle nous permet de parler de deux suffixes différents: $-\operatorname{ur}(\breve{a})$ et -ătur(ă), bien qu'ils aient la même signification. Un autre exemple du résultat de l'absorption morphologique est le suffixe -ir(e) qui, combiné avec des bases substantives, crée des noms abstraits, cf. :

$$
\begin{aligned}
& i u b i_{\mathrm{VB}} \text { 'aimer' } \rightarrow \text { iubi-r } r(e)_{\mathrm{SF}} \text { 'amour' } \\
& \operatorname{om}_{\mathrm{SM}}\left(\text { oámeni } i_{\mathrm{NOM} . \mathrm{PL}} \text { ' 'homme' } \rightarrow \text { omen-ir }(e)_{\mathrm{SF}}\right. \text { 'humanité' }
\end{aligned}
$$

Dumneze $(u)_{\mathrm{SM}}$ 'Dieu' $\rightarrow$ dumneze-ir $(e)_{\mathrm{SF}}$ 'divinité'

\section{Alternants phonologiques qualitatifs}

Les alternances conditionnées phonologiquement, bien sûr, n'ont pas nécessairement le caractère uniquement quantitatif. Il semble que les alternances qualitatives soient beaucoup plus fréquentes ${ }^{10}$. Leur nature phonologique ne signifie pas qu'elles sont toujours obligatoires. Quelques exemples de ces alternances ont été présentés ci-dessus (roum. ecologie $\mathrm{SF} \rightarrow$ ecolog $_{\mathrm{SM}}$ ), mais il est possible d'en trouver d'autres qui ne se réfèrent qu'à seules les alternances qualitatives :

roum. muncitor $(\varnothing)_{\mathrm{SM}}$ 'ouvrier' $\rightarrow$ muncit $[$ oa $] r(e)_{\mathrm{SF}}$ 'ouvrière' roum. profesor $\left(\varnothing_{\mathrm{SM}}\right.$ 'professeur' $\rightarrow$ profes $[$ oa $] r(\breve{a})_{\mathrm{SF}}$ 'professeure' roum. psiholog $(\varnothing)_{\mathrm{SM}}$ 'psychologue' $\rightarrow$ psihol[oa $] g(\breve{a})$ ou $p \operatorname{siholog}(\breve{a})_{\mathrm{SF}}$ 'idem (féminin)' pol. królik $(\varnothing)_{\mathrm{SM}}$ 'lapin' $\rightarrow$ króli $[c z]-\operatorname{arni}(a)$ ou królik-arni $(a)_{\mathrm{SF}}$ 'clapier'

Aux alternants quantitatifs et qualitatifs, membres des alternances décrites cidessus, en raison de leur conditionnement phonologique et de leur occurrence nonobligatoire, nous attribuons le statut de formants secondaires, c'est-à-dire de tels formants qui ne sont qu'auxiliaires des formants primaires - exposants proprement dits de changements sémantiques du dérivé par rapport à la base. Dans les formations analysées plus haut, ce sont les formants suffixaux ou les formants paradigmatiques qui sont primaires.

En polonais, cependant, ce ne sont pas toutes les alternances qui ont seulement la fonction secondaire. Les alternances qualitatives qui ne sont pas conditionnées phonologiquement et dont les membres sont les exposants de changements de sens

\footnotetext{
${ }^{10}$ C'est-à-dire les alternances dont aucun de deux membres n'est zéro.
} 
(ou, plus précisément, les exposants de changements pragmatiques), constituent des exemples d'apophonie ${ }^{11}$. L'apophonie, se produisant dans la dérivation des augmentatifs et des diminutifs, consiste d'habitude en un changement de la consonne finale du thème de base, cf. :

mięs $(o)_{\mathrm{SF}}$ 'viande' $\rightarrow$ mię $[c h](o)_{\mathrm{SF}}$ 'idem (augmentatif)'

papieros $(\varnothing)_{\mathrm{SM}}$ 'cigarette' $\rightarrow$ papiero $[$ ch $](\varnothing)_{\mathrm{SM}}$ 'idem (augmentatif)'

kietbas $(a)_{\mathrm{SF}}$ 'saucisse' $\rightarrow$ kietba $[$ ch $](a)_{\mathrm{SF}}$ 'idem (augmentatif)'

Des alternances pareilles peuvent également accompagner un formant négatif ou paradigmatique et avoir le caractère quantitatif, cf. :

$k l u s-k(a)_{\mathrm{SF}}$ 'nouille' $\rightarrow k l u[c h]-\varnothing-(a)_{\mathrm{SF}}$ 'idem (augmentatif)'



ćwiart-k(a) $)_{\mathrm{SF}}$ 'quart' $\rightarrow$ ćwiar $[\varnothing]-\varnothing-(a)_{\mathrm{SF}}$ 'idem (augmentatif)'

Il est possible que les formants résultant de l'apophonie n'existent pas en roumain.

\section{Formants négatifs}

À la suite de l'attribution aux alternants quantitatifs du statut de formants de type distinct, le formant négatif, lui, va être défini de manière étroite, c'est-à-dire comme un effet de la soustraction à la base dérivationnelle d'un morphème qui fait partie de sa structure ${ }^{12}$. Voici les exemples roumains et polonais :

roum. botez- $a_{\mathrm{VB}}$ 'baptiser' $\rightarrow$ botez- $Ø(\varnothing)_{\mathrm{SN}}$ 'baptême'

roum. $z$ bur- $a_{\mathrm{VB}}$ 'voler' $\rightarrow$ zbor- $-(\varnothing)_{\mathrm{SN}}$ 'vol'

roum. se obosi $i_{\mathrm{VB}}$ 'se fatiguer' $\rightarrow \varnothing$ obosi $i_{\mathrm{VB}}$ 'fatiguer'

pol. $\operatorname{ciesz}\left(y c\right.$ ć) $s i e_{\mathrm{VB} . \mathrm{INTR}}$ 'se réjouir' $\rightarrow \operatorname{ciesz}\left(y c\right.$ c) $\varnothing_{\mathrm{VB} . \mathrm{TR}}$ 'réjouir'

pol. ogór-ek $(\varnothing)_{\mathrm{SF}}$ 'concombre' $\rightarrow$ ogór-Ø(Ø) $)_{\mathrm{SM}}$ 'idem (augmentatif)'

pol. purpur-ow $(y)_{\mathrm{ADJ}}$ 'pourpre' $\rightarrow \operatorname{purpur}(a)_{\mathrm{SF}}$ 'couleur pourpre'

Dans le troisième et le quatrième exemple, nous constatons l'occurrence d'un formant négatif extrafixal, dans les autres - l'occurrence d'un formant négatif suffixal ( $-a$ dans les verbes roumains est un suffixe thématique).

Dans certains cas, le formant négatif est un co-formant accompagnant l'affixation :

roum. evoluțion-ism $m_{\mathrm{SN}}$ 'évolutionnisme' $\rightarrow$ evoluțion- $\varnothing$-ist $(\varnothing)_{\mathrm{SM}}$ 'évolutionniste' pol. komun-izm ${ }_{\mathrm{SN}}$ 'communisme' $\rightarrow$ komun- $\varnothing$-ist $(a)_{\mathrm{SM}}$ 'communiste' roum. se indoi $i_{\mathrm{VB}}$ 'douter' $\rightarrow \varnothing$ indo-ial $(\breve{a})_{\mathrm{SF}}$ 'doute'

\footnotetext{
${ }^{11}$ La notion d'apophonie que nous introduisons englobe aussi les alternances consonantiques.

${ }^{12}$ Notre interprétation est une modification de la conception de Wróbel (2001, pp. 174-178), cité par Kaproń-Charzyńska (2005, pp. 14).
} 
pol. wy-mien $(i c)_{\mathrm{VB}}$ 'échanger' $\rightarrow z a-\varnothing$-mien $(i c ́)_{\mathrm{VB}}$ 'échanger une chose contre une autre' roum. $\operatorname{acord}(\varnothing)_{\mathrm{SN}}$ 'accord' $\rightarrow$ con- $\varnothing-$ cord- $a_{\mathrm{VB}}$ 'concorder' pol. Ukraini-ec $(\varnothing)_{\mathrm{SM}} /$ ukraincts/ 'Ukrainien' $\rightarrow$ Ukrain- $\varnothing-k(a)_{\mathrm{SF}}$ 'Ukrainienne, 13

La dérivation négative ainsi comprise est le contraire exact de l'affixation, ce qui nous permet de distinguer un ensemble théorique complet de sous-types de formants négatifs, parallèle à l'ensemble d'affixes, bien que probablement seulement trois d'entre eux soient réalisés en polonais et en roumain : le formant négatif extrafixal, le formant négatif suffixal et le formant négatif préfixal.

\section{Formants paradigmatiques}

Puisque la formation des mots analyse les relations qui unissent les lexèmes, et non seulement celles qui unissent leurs formes de base, les deux membres de la relation dérivationnelle sont les unités lexicales, chacune comprenant un ensemble complet de ses formes flexionnelles. Les désinences qui suivent un suffixe ne font pas partie de cet ensemble, car elles sont des morphèmes distincts. De plus, un seul et même suffixe peut avoir la capacité d'adhésion à de différents ensembles de désinences, aussi dans les cas où il réalise toujours la même catégorie dérivationnelle, c'est-à-dire quand il a une seule et même signification, comme dans les exemples roumains suivants :

$\operatorname{apăra~}_{\mathrm{VB}}\left(\right.$ apărând $\left._{\mathrm{PTCP} . \mathrm{ACT}}\right)$ 'protéger' $\rightarrow$ apăr-ătoar $(e)_{\mathrm{SF}}$ 'protection (objet)' $s c h i m b a_{\mathrm{VB}}\left(\right.$ schimbând $\left.d_{\mathrm{PTCP} . \mathrm{ACT}}\right)$ 'changer' $\rightarrow$ schimb-ător $(\varnothing)_{\mathrm{SN}}$ 'transmission (mécanisme)'

La suffixation est accompagnée ici d'un formant paradigmatique que nous définissons comme un résultat du changement de paradigme flexionnel du dérivé par rapport à la base. Dans les paires dérivationnelles ci-dessus, nous distinguons le sous-type de ce formant : le formant extraparadigmatique, c'est-à-dire celui qui change non seulement l'ensemble de désinences, mais aussi l'ensemble de catégories flexionnelles disponibles. Tout à côté de ce dernier, il est possible de distinguer le formant intraparadigmatique, c'est-à-dire un formant qui ne change que l'ensemble de désinences, sans affecter l'ensemble de catégories. En roumain, cela se rapporte à des exemples tels que :

$$
\begin{aligned}
& \operatorname{profesor}(\varnothing \sim i)_{\mathrm{SM}} \rightarrow \operatorname{profesoar}(\breve{a} \sim e)_{\mathrm{SF}} \\
& \operatorname{rând}(\varnothing \sim u r i)_{\mathrm{SN}} \text { 'rang (série)' } \rightarrow \operatorname{rânduleț(}(\varnothing \sim e)_{\mathrm{SN}} \text { 'idem (diminutif)' }
\end{aligned}
$$

Dans d'autres cas, les désinences elles-mêmes restent intactes, mais c'est l'ensemble de catégories flexionnelles qui change :

\footnotetext{
${ }^{13}$ Nos exemples polonais sont empruntés à Nagórko (2010, pp. 198-199). Dans le dernier, on observe en plus l'alternance $/ \mathrm{n} /: / \mathrm{n} /$ qui se produit.
} 
roum. medi $(u)_{\mathrm{ADJ}}\left(\operatorname{medi}(e)_{\mathrm{F}}\right)$ 'moyen' $\rightarrow$ medi $(e)_{\mathrm{SF}}$ 'moyenne (grandeur)' roum. fungicid $(\varnothing)_{\mathrm{ADJ}}$ 'fongicide' $\rightarrow$ fungicid $(\varnothing)_{\mathrm{SN}}$ '(produit) fongicide' roum. $\operatorname{român}(\varnothing)_{\mathrm{SM}}$ 'Roumain' $\rightarrow \operatorname{român}(\varnothing)_{\mathrm{ADJ}}$ 'roumain' pol. znajomy $_{\mathrm{ADJ}}$ 'connu' $\rightarrow$ znajomy $_{\mathrm{SM}}$ 'connaissance (personne)'

Le formant extraparadigmatique ne signifie pas nécessairement un changement de partie du discours. Analysons les paires dérivationnelles suivantes :

roum. $i t ̦(\breve{a})_{\mathrm{SF} . \mathrm{SG}}\left(i t ̦(e)_{\mathrm{PL}}\right)$ 'lice (aiguille)' $\rightarrow i t ̦-\operatorname{ar}(i)_{\mathrm{SM} . \mathrm{PL}-\mathrm{TANT}}$ 'type de pantalon traditionnel' roum. ochelar $(i)_{\mathrm{SM} . \mathrm{PL}-\mathrm{TANT}}$ 'lunettes' $\rightarrow$ ochelar-ist $(\varnothing)_{\mathrm{SM} . \mathrm{SG}}\left(\right.$ ochelarișt $\left.(i)_{\mathrm{PL}}\right)$ 'binoclard' roum. $\operatorname{brânz}(\breve{a})_{\mathrm{SF} . \mathrm{SG}-\mathrm{TANT}}$ 'fromage' $\rightarrow$ brânz-et $(\text { uri })_{\mathrm{SN} . \mathrm{PL}-\mathrm{TANT}}$ 'types de fromages' pol. $C z e c h(y)_{\mathrm{NP.SN}-\mathrm{M} . \mathrm{PL}-\mathrm{TANT}}$ 'Tchéquie' $\rightarrow \operatorname{Czech}(\varnothing)_{\mathrm{SM} . \mathrm{SG}}\left(C z e s(i)_{\mathrm{PL}}\right)$ 'Tchèque (personne)' pol. $c z y t a\left(c^{\prime}\right)_{\mathrm{VB} . I P F V}$ 'lire' $\rightarrow$ prze-czyta $(\dot{c})_{\mathrm{VB} . \mathrm{PFV}}$ 'idem'

On n'observe pas de changement de partie du discours ni de fonction syntaxique propre au lexème dans aucune de ces paires dérivationnelles. Et pourtant, il y a un changement dans l'ensemble de catégories flexionnelles : en polonais, le substantif Czechy ne connaît pas la catégorie grammaticale du nombre, et le verbe appartient à la classe des perfectifs qui ne forment pas le futur composé dans cette langue.

\section{Formant zéro}

En roumain, il existe des paires dérivationnelles qui se caractérisent par un changement de fonction syntaxique du dérivé par rapport à la base (parfois accompagné d'un changement de sens) sans exposants formels de dérivation. Cela se produit, entre autres, lorsque la base dérivationnelle et le dérivé sont invariables et n'ont donc pas de paradigme flexionnel. Considérons l'exemple :

$$
\text { gata }_{\mathrm{ADJ}} \text { 'prêt' } \rightarrow \text { gata }_{\mathrm{ADV}} \text { 'étant prêt' }
$$

Le mot gata fonctionne comme adverbe, par exemple dans les phrases ci-dessous :

Fiecare nouă generație, pășind în viață, găsește ${ }_{\mathrm{VB}}$ gata $_{\mathrm{ADV}}$ pregătite forțele de producție (...) (DLRLC, 1955-57).

În fundul sufletului lui însă stă ${ }_{\mathrm{VB}}$ gata $_{\mathrm{ADV}}$ hotărârea (DEXI).


1955-57).

Cela signifie que la fonction syntaxique des formants dérivationnels - en l'absence de changement de sens - se réalise ici, mais qu'elle n'a pas d'exposant positif $^{14}$. Puisque l'exposant zéro est considéré un phénomène « normal » dans la 2005).

${ }^{14}$ Notre interprétation s'appuie en gros sur la description de la dérivation zéro en anglais (cf. Bauer, 
flexion, il peut donc y être emprunté pour les besoins de la formation des mots et nommé formant zéro. À condition, toutefois, qu'il soit au moins l'exposant de changement de propriétés du lexème non-identifiables dans sa forme, c'est-à-dire l'exposant de changement de ses propriétés syntaxiques. Un changement de ces propriétés peut être accompagné d'un changement de sens. En même temps, il convient de noter que ce ne sont pas toutes les modifications des propriétés fonctionnelles du dérivé par rapport à la base dérivationnelle qui ont le caractère de changement de partie du discours, ce qui est bien visible dans les paires dérivationnelles de verbes intransitifs et transitifs, comme dans l'exemple polonais :

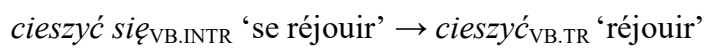

Dans la paire dérivationnelle ci-dessus les propriétés syntaxiques changent, mais non pas la partie du discours. Le premier verbe, bien que formellement plus complexe, est sémantiquement plus simple, ce qui détermine la direction de la motivation.

Dans les exemples de paires dérivationnelles polonaises trouvés par l'auteur c'est l'extrafixe się qui est l'exposant d'intransivité. En roumain, ce sont les paires homonymes de verbes transitifs et intransitifs qui sont pertinents dans ce cas. Voyons l'exemple d'une telle paire avec les définitions lexicographiques des significations distinctes du verbe asurzi :

1. vb.intr 'a-și pierde auzul' (fr. : 'perdre l'ouïe')

2. $v b . t r$ 'a face pe cineva să-și piardă auzul' (fr. : 'faire perdre l'ouïe à quelqu'un') ; (MDA, 2010)

Il est facile à constater qu le verbe transitif (2.) est sémantiquement plus complexe. D'autre part, les deux verbes sont identiques dans toutes leurs formes et ont le même ensemble de catégories flexionnelles, de sorte que l'exposant de dérivation est ici zéro :



En voici d'autres exemples en roumain :

$r o b i_{\mathrm{VB} . \mathrm{INTR}}$ 'être un esclave' $\rightarrow r o b i_{\mathrm{VB} . \mathrm{TR}}$ 'esclavager'

odihni $i_{\mathrm{VB} . \mathrm{INTR}}$ 'se reposer' $\rightarrow$ odihni $i_{\mathrm{VB} . \mathrm{TR}}$ 'reposer'

\section{Formant accentuel}

Pendant que la langue polonaise, à quelques exceptions près, se caractérise par l'accent paroxytonique fixe, en roumain, l'accent peut tomber sur des syllabes différentes du mot et avoir la valeur distinctive. En ce qui concerne la déclinaison, 
l'accent roumain y est fixe, tandis que dans la conjugaison, il est mobile. Le phénomène de déplacement d'accent peut être observé aussi dans la dérivation, cf. :

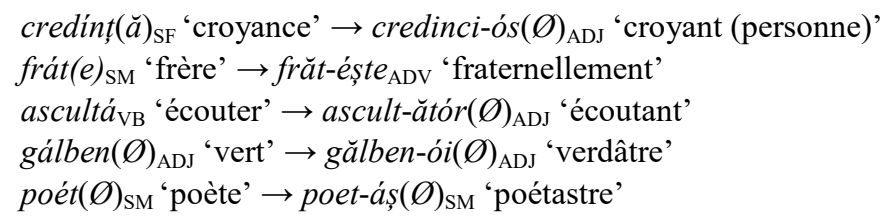

Comme on peut l'observer, l'omission dans l'analyse du déplacement d'accent sur le suffixe conduirait à une fausse description de certains d'entre les dérivés cités : *credíncios, etc. Cela nous permet de distinguer un autre type de formant : le formant accentuel.

Le formant accentuel (appelé aussi "prosodique ») est identifié en polonais dans certains types de mots composés, tels que wiary SF.GEN.SG godny $_{\mathrm{ADJ}}$ 'digne de con-

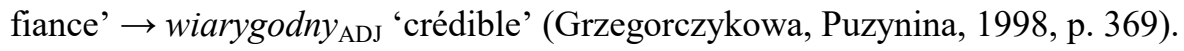

\section{CONCLUSION}

Les types de formants et de thèmes dérivationnels présentés dans cet article ne constituent certainement pas une liste exhaustive. La détermination d'un ensemble complet de ces types ainsi que d'un ensemble de types de structures formelles des dérivés (ce dernier s'appuyant sur le premier) nécessite des recherches plus approfondies. Cependant, l'analyse faite ici prouve que, par exemple, pour pouvoir formuler des modèles corrects de telles structures, il est nécessaire de prendre en compte les types de thèmes dérivationnels. Parmi les applications possibles de notre analyse, on peut prévoir de futures recherches contrastives aussi bien qu'une possibilité d'élaborer les modèles formels des nids dérivationnels en roumain à l'aide des propriétés formelles des dérivés.

\section{APPENDICE}

Abréviations spécifiques utilisées dans l'article ${ }^{15}$

$\begin{array}{ll}\text { ACT } & \text { actif } \\ D & \text { dérivé } \\ \mathrm{F}_{\text {SUF }} & \text { suffixe } \\ \mathrm{F}_{\mathrm{PAR}} & \text { formant paradigmatique }\end{array}$

\footnotetext{
${ }^{15}$ Conformes aux gloses de Leipzig (The Leipzig Glossing Rules) et aux normes employées par les dictionnaires roumains courants (DEXI, DLRLC...).
} 


$\begin{array}{ll}\mathrm{F}_{\mathrm{ALT}} & \text { alternant } \\ \mathrm{F}_{\mathrm{ACC}} & \text { formant accentuel } \\ \mathrm{INV} & \text { invariable } \\ \mathrm{NP} & \text { nom propre } \\ \mathrm{PL}-\mathrm{TANT} & \text { plurale tantum } \\ \mathrm{R} & \text { thème (de la base dérivationnelle) } \\ \mathrm{R}_{\mathrm{CURT}} & \text { thème abrégé } \\ \mathrm{R}_{\mathrm{SEC}} & \text { thème secondaire } \\ \mathrm{R}_{\mathrm{EXT}} & \text { thème élargi } \\ \mathrm{R}_{\mathrm{RED}} & \text { thème réduit } \\ \mathrm{SG}-\mathrm{TANT} & \text { singulare tantum } \\ \mathrm{SN}-\mathrm{M} & \text { substantif non-masculin-personnel }\end{array}$




\section{BIBLIOGRAPHIE}

Apresjan, J.D. (2000). Semantyka leksykalna. Synonimiczne środki języka. Wrocław: Zakład Narodowy im. Ossolińskich.

Avram, M., Carabulea, E., Ciobanu, F. (éd.) (1978). Formarea cuvintelor în limba română. Vol. II. Prefixele. Bucureşti : Editura Academiei R.S.R.

Ciobanu, F., Hasan, F. (1970). Formarea cuvintelor în limba română. Vol. I. Compunerea. București : Editura Academiei R.S.R.

Coteanu, I. (2007). Formarea cuvintelor în limba română. Bucureşti : Editura Universităţii din Bucureşti.

Cychnerski, T. (1999). Fleksja werbalna w języku rumuńskim. Poznań : Wydawnictwo Naukowe UAM.

DEXI, (2007) = Dima, E. (éd.) (2007). Dicţionar explicativ ilustrat al limbii române. Chişinău : Editura ARC \& Editura GUNIVAS.

DLRLC, (1955-57) = Rosetti, A. (éd.) (1955-1957). Dictionarul limbii romîne literare contemporane. Vol. I-IV. București : Editura Academiei Republicii Populare Romîne.

Dunaj, B. (2007). Wspótczesny słownik języka polskiego. Warszawa: Langenscheidt.

Grzegorczykowa, R., Puzynina, J. (1998). Słowotwórstwo. Problemy ogólne słowotwórstwa. In R. Grzegorzykowa, R. Laskowski, H. Wróbel (éd.). Gramatyka wspótczesnego języka polskiego. Morfologia. Warszawa: Wydawnictwo Naukowe PWN, pp. 361-388.

Guţu Romalo, V. (éd.) (2008). Gramatica limbii române. I. Cuvântul. Bucureşti : Editura Academiei Române.

Jadacka, H. (2003). Zagadnienie motywacji słowotwórczej w opisie gniazdowym. In M. Skarżyński (éd.). Stowotwórstwo gniazdowe. Historia, metoda, zastosowania. Kraków: Księgarnia Akademicka.

Jaroszewicz, H. (2016). Derywacja frazeologiczna w języku polskim i serbskim. Wrocław: Wydawnictwo Uniwersytetu Wrocławskiego.

Kaproń-Charzyńska, I. (2005). Derywacja ujemna we wspótczesnym języku polskim. Rzeczowniki i przymiotniki. Toruń: Wydawnictwo Top Kurier.

Kreja, B. (1996). Studia z polskiego stowotwórstwa. Gdańsk: Wydawnictwo UG.

Kuryłowicz, J. (1936). Dérivation lexicale et dérivation syntaxique. In Bulletin de la Societé Linguistique de Paris. 37, pp. 79-92.

Laskowski, R. (1998). Zagadnienia ogólne morfologii. In R. Grzegorzykowa, R. Laskowski, H. Wróbel (éd.). Gramatyka wspótczesnego języka polskiego. Morfologia. Warszawa: Wydawnictwo Naukowe PWN, pp. 27-86.

MDA, (2010) = Sala, M., \& Dănăilă, I. (éd.) (2010). Mic dicționar academic. Vol. I-II. București : Univers Enciclopedic Gold.

Mugdan, J. (2015). Units of word-formation. In P.O. Müller, I. Ohnheiser, S. Olsen, F. Rainer (éd.). Word-Formation. An International Handbook of the Languages of Europe. Vol. 1. Berlin : De Gruyter Mouton, pp. 235-301. DOI : 10.1515/9783110246254-017.

Müller, P.O., Ohnheiser, I., Olsen, S., Rainer, F. (éd.) (2015). Word-Formation. An International Handbook of the Languages of Europe. Vol. 1. Berlin : De Gruyter Mouton. DOI : 10.1515/9783110246254.

Nagórko, A. (2010). Podręczna gramatyka języka polskiego. Warszawa: Wydawnictwo Naukowe PWN.

Negraru, M. (éd.) (1971). Formarea cuvintelor în limba română. Cercetare bibliografică. București : Biblioteca Centrală Universitară.

Rădulescu Sala, M. (éd.) (2015). Formarea cuvintelor în limba română. Vol. IV. Sufixele. Derivarea nominală şi adverbială. Partea 1. Bucureşti : Editura Academiei Române.

Skarżyński, M. (1999). Powstanie i rozwój polskiego stowotwórstwa opisowego. Kraków: Universitas.

Skarżyński, M. (éd.) (2003). Stowotwórstwo gniazdowe. Historia, metoda, zastosowania. Kraków: Księgarnia Akademicka. 
Skarżyński, M. (éd.) (2004). Słownik gniazd słowotwórczych współczesnego języka ogólnopolskiego. Vol. 3. Gniazda odczasownikowe (part II). In H. Jadacka, Stownik gniazd słowotwórczych wspótczesnego języka ogólnopolskiego. Kraków: Towarzystwo Wydawnicze „Historia Iagellonica”.

Szymanek, B. (2015). A Panorama of Polish Word-Formation. Lublin: Wydawnictwo KUL.

The Leipzig Glossing Rules. Leipzig: Max Planck Institute for Evolutionary Anthropology. Department of Linguistics, https://www.eva.mpg.de/lingua/resources/glossing-rules.php.

Vasiliu, L. (1989). Formarea cuvintelor în limba română. Vol. III. Sufixele. Derivarea verbală. București : Editura Academiei Române.

Vater, H. (2015). Wprowadzenie do lingwistyki. Wrocław: Oficyna Wydawnicza Atut.

Viaro, M.E, Bizzocchi, A.L. (2016). Proposal of new concepts and a new notatnion in formulating etymological propositions and discussions. In Alfa : Revista de Lingüistica. 60, pp. 587-608. DOI : 10.1590/1981-5794-1612-6.

Vogelgesang, T. (2001). Słownik gniazd słowotwórczych współczesnego języka ogólnopolskiego. Vol. 1: Gniazda odprzymiotnikowe. In H. Jadacka, Stownik gniazd stowotwórczych wspótczesnego języka ogólnopolskiego. Kraków: Universitas.

Wróbel, H. (2001). Gramatyka języka polskiego. Kraków: Od Nowa.

Zołotowa, W.S. (1962). Rzeczowniki dewerbalne osobowe we współczesnej polszczyźnie. In Poradnik Językowy. Z. 7-8, pp. 319-320. 